\title{
UNE ONTOLOGIE DE LA CULTURE DE LA VIGNE : DES SAVOIRS ACADÉMIQUES AUX SAVOIRS D'EXPÉRIENCE
}

\author{
Clémentine Hugol-Gential ${ }^{1}$, Marie Simon ${ }^{2}$, Aurélie BertauX ${ }^{3}$, \\ Ouassila LABBANi NARSis ${ }^{4}$, Rami BelKaroui 5 , \\ Amira MOUAKHER ${ }^{6}$ et Christophe NiCOLlE ${ }^{7}$
}

\begin{abstract}
Dans le cadre d'un projet FUI initié en octobre 2016 (projet winecloud $^{8}$ ) visant à construire un outil de traçabilité et prédictif du cycle de la vigne et du vin, un travail sur la collecte et la nature des savoirs a été nécessaire de manière à penser un système ontologique qui se rapproche le plus du raisonnement du domaine métier. Le présent article vise plus spécifiquement à étudier le cycle de vie de la vigne. Nous rendons compte que les savoirs
\end{abstract}

1 Clémentine Hugol-Gential est maîtresse de conférences à l'Université de Bourgogne, Franche- Comté, Laboratoire CIMEOS Lab (EA 4177).

2 Marie Simon est attachée à l'Université de Bourgogne Franche-Comté et ingénieure d'études.

3 Aurélie Bertaux est maîtresse de conférences à l'Université de Bourgogne FrancheComté, Laboratoire CIAD (EA7533).

4 Ouassila Labbani Narsis est maîtresse de conférences à l'Université de Bourgogne Franche- Comté, Laboratoire CIAD (EA7533).

5 Rami Belkaroui est post-doctorant à l'Université de Bourgogne Franche Comté, Laboratoire CIAD (EA7533).

6 Amira Mouakher est post-doctorante à l'Université de Bourgogne Franche Comté, Laboratoire CIAD (EA7533).

7 Christophe Nicolle est professeur des Universités à 1'Université de Bourgogne Franche Comté, Laboratoire CIAD (EA7533).

8 https://www.vitagora.com/vitagora/actus-media/foodtech-innovation-projets-fui-3dprinting-vin/. Pour voir les éléments relatifs au projet : http://winecloud. checksem.fr/presentation

Recherches en communication, $\mathrm{n}^{\circ} 48$ - Article publié le 30/042019 
académiques présents dans les sources théoriques et scientifiques s'ajustent, se réactualisent à la lumière des savoirs d'expérience des viticulteurs. Ce travail s'attache également à analyser la nature protéiforme des savoirs d'expérience et à rendre compte de leur pluralité.

\section{Introduction}

Le projet de recherche dont les données sont issues a pour objectif de développer la toute première plateforme sur les chaînes de valeur viti et vinicole en se basant sur des données issues de la cave de Lugny, coopérative partenaire du projet en Saône-etLoire (71). Cette plateforme a pour objet de combiner des données issues de capteurs (Internet des Objets) avec des connaissances métiers pour construire des services innovants. Celle-ci, dotée d'outils logiques et sensibles au contexte, sera en premier lieu utile pour les viticulteurs : prédiction de l'état des vignes et recommandations pour les pratiques de production afin de permettre une production de vin plus raisonnée. Système ouvert, professionnels et grand public y trouveront des conseils et informations adaptés à leur propre utilisation (viticulture, œnologie, consommation...). En proposant, une traçabilité complète du cycle du vin, de la vigne aux verres du consommateur, cette plateforme qui sera ensuite accessible aux viticulteurs permettra par ailleurs de renforcer le lien entre professionnels du vin, cavistes et consommateurs.

Nous souhaitons ici rendre compte de l'usage des méthodologies mobilisées en Sciences humaines et sociales et en Informatique afin de construire une ontologie concernant le cycle de la vigne. Ce travail ontologique est constitué à partir de la captation de quatre types de données :

- Données brutes issues de capteurs simples (température, taux de $\mathrm{CO}_{2}$, etc.) au pied des vignes (fournissent des paramètres contextuels).

- Données traitées issues de capteurs intelligents (caméras embarquées avec algorithmes de traitement d'images). 
- Données brutes issues de capteurs intégrés à la cuve (fournissent des paramètres physiques tels que la température, le $\mathrm{pH}$, la turbidité, etc.)

- Corpus de références théoriques et scientifiques ainsi que des verbatim professionnels issus de la conduite d'entretien analysés de manière à rendre compte de leurs complémentarités, apports et oppositions.

Dans le cadre de cet article, un focus est proposé sur la méthode employée afin de capter les savoirs des professionnels qui peuvent être à la fois scientifiques et théoriques mais également actualisés et modifiés en prise à la réalité du terrain (savoirs d'expérience). Il s'agit dès lors de s'intéresser aux savoirs académiques mobilisés dans les champs scientifiques et théoriques et de voir leur actualisation, modification, circulation au sein des savoirs d'expérience et pratiques professionnelles. Ainsi, plusieurs sources ont été investiguées grâce à une veille documentaire et à la conduite d'entretiens avec vingt viticulteurs : les documents de référence la plupart du temps de nature académique (théoriques, scientifiques, voire législatifs) qui sont complétés par les savoirs d'expérience des viticulteurs (collectés par la conduite d'entretiens). Il ne s'agit donc pas d'opposer les différentes formes de savoir, mais de s'interroger par rapport au processus de transformation et d'ajustement, pouvant impacter les pratiques et d'étudier spécifiquement la complémentarité des savoirs afin de créer une ontologie se rapprochant du raisonnement métier. Ainsi, nous présenterons dans un premier temps ce qu'est une ontologie et quels sont les enjeux méthodologiques de son application au cycle de la vigne et du vin. Dans un second temps, nous exposerons la création d'un corpus utilisé afin de collecter les savoirs théoriques et scientifiques. Enfin, nous expliciterons la méthode employée dans le but de collecter les savoirs d'expérience des viticulteurs.

\section{L'ontologie : objet de mise en relation logique des savoirs}

Depuis Aristote, l'humain s'est demandé comment représenter le monde qui l'entoure en étudiant ses propriétés et catégories, 
cette science s'appelle la métaphysique. Cette étude a été l'une des sources d'inspiration pour l'intelligence artificielle qui a vu se développer les réseaux sémantiques (Quillian, 1968) et les frames (Minsky, 1974 ; Sowa, 1984) afin d'encoder des connaissances taxonomiques. Leurs descendants, les ontologies, sont aujourd'hui au cœur de l'étude des systèmes d'information et du Web. Dans le cadre du projet Winecloud, nous considérons la définition proposée par Studer (1998) pour qui « une ontologie est définie comme "a formal, explicit specification of a shared conceptualization" ". Dans ce contexte, le développement d'une ontologie est un processus dynamique et incrémental débutant par la création d'une ontologie brute qui a besoin d'être révisée, raffinée et complétée. Ce cycle est appelé cycle de vie de l'ontologie.

Dans notre projet, nous présentons une nouvelle approche basée sur une ontologie des événements afin de permettre une interprétation métier des remontées de capteurs. L'ontologie créée vise donc à corréler des données issues du processus agricole afin de développer un système de traçabilité des outils prédictifs (notamment pour l'apparition de maladies, de ravageurs, de risques climatiques, etc.), optimiser si besoin les processus de travail de la vigne (traitements phytosanitaires, insecticides, etc.) et du vin (ajout de sulfites, contrôle de la fermentation alcoolique, etc.) tout en fournissant une connaissance qualitative (données issues des entretiens et de l'ontologie) et quantitative (données issues des capteurs) des paramètres d'influence pour mieux contrôler la production. Le traitement des données se fait par des méthodes de data mining, autrement dit des méthodes de fouilles terminologiques permettant de faire émerger des connaissances implicites interprétées et identifiées par la mise en exergue de leurs relations (relation de corrélation). C'est un processus d'Analyse Formelle de Concepts qui nous fournira les corrélations recherchées. Par la suite, il est possible de se baser sur des algorithmes d'exploration de données et de générer des corrélations. Ces connaissances sont ensuite sémantiquement qualifiées au sein d'une ontologie. Il s'agit donc de développer un système intelligent de traçabilité logique du cycle de vie de la vigne et de la vinification à destination des viticulteurs, des viniculteurs et d'autres partenaires 
externes (exemple : Institut français du Vin', Chambre d'Agriculture $^{10}$ ). Les résultats des différents protocoles d'études mis en place sont utilisés pour construire une représentation sémantique du domaine en utilisant les technologies du Web Sémantique. Une ontologie décidable est développée en logique de description sur les connaissances (savoir-faire) liées au domaine vitivinicole. Une ontologie est dite décidable s'il existe un procédé de calcul (algorithme) permettant de déterminer si une règle est une conséquence sémantique de l'ontologie ou non. Ce travail de formalisation de l'ontologie permet alors de construire des règles de raisonnement logique permettant de représenter une connaissance métier. À partir des données remontées par les capteurs et de données environnementales, ces règles permettront de fournir un raisonnement proche de celui du domaine métier et de construire des relations de causalité. L'ontologie par ailleurs se nourrit de l'analyse sémantique qui permet ensuite d'agencer tout un ensemble de règles logiques permettant de donner des explications qui justifient que le résultat annoncé est valide, mais c'est aussi un objet de médiation des savoirs au sens entendu dans les Sciences de l'Information et de la Communication. L'ontologie devient un objet donnant lieu à des usages technologiques, ainsi : «la notion de médiation semble donc désigner, en ce cas, les opérations - ainsi que leurs effets - de technicisation du processus de communication (médiation technique) et, en même temps, de l'intervention de la dimension subjective dans les pratiques de communication (médiation sociale) » (Davallon, 2003, p. 43). Ainsi, à partir de l'ontologie peuplée, nous avons réalisé des inférences (i.e. raisonnements) entre par exemple les dates, les capteurs météorologiques et l'apparition des maladies ${ }^{11}$. Ces raisonnements permettent de proposer des services aux utilisateurs, en particulier les viticulteurs, tels que rechercher des événements qui se produisent sur la même période, identifier les causes d'un événement ou rechercher des événements ayant une cause particulière.

9 http://www.vignevin.com

$10 \mathrm{https}: / /$ chambres-agriculture.fr

11 Une démonstration est visible ici : http://winecloud.checksem.fr/explain-cycle-vigne 


\section{La mise en œuvre d'un système de traçabilité du cycle de la vigne : les enjeux d'une méthodologie pluridisciplinaire}

Pour développer un système ontologique de traçabilité, il est indispensable, en amont, de capter les savoirs incorporés et intériorisés des vitiviniculteurs. S'il existe des normes agronomiques en vigueur, un savoir théorique, scientifique et législatif régissant les règles de viticulture et de viniculture, des savoirs métiers se construisent également in situ, dans l'action en prise avec les réalités de terrain. Ces savoirs reposent sur l'expérience, la pratique, l'observation, l'essai, mais aussi l'erreur (Richardson, 2005 ; Sylvestre, 1997). Se confrontent alors les savoirs théoriques et les savoirs en prise avec la réalité métier (les savoirs d'expérience). Si les premiers permettent de saisir les contours théoriques et scientifiques de la mise en culture de la vigne et de la vinification, les seconds soulignent les bricolages, les détournements, les adaptations des normes et des règles mis en œuvre par les acteurs pour s'adapter à des contextes logistiques, climatiques ou autres. Par ailleurs, certains savoirs sont transmis uniquement entre les viticulteurs et viniculteurs reposant donc sur une culture orale. Ainsi les savoirs métiers sont pluriels, issus de l'apprentissage académique, de la transmission entre pairs, de l'appréhension et de la connaissance terrain.

Afin de saisir ces savoirs métiers dans toute leur complexité, il convient alors de développer une démarche inductive avec en premier lieu la constitution d'un corpus permettant de collecter les savoirs théoriques et scientifiques indispensables (référentiels) concernant la culture de la vigne et les méthodes de vinification. Dans un second temps, afin de confronter la théorie et la réalité pratique et dans l'objectif de mieux appréhender le quotidien des vitiviniculteurs et des professionnels du vin, il est indispensable de mettre en œuvre une observation participante afin de saisir au mieux "les impondérables de la vie quotidienne » (Malinowski 1963 [1922] : 63). De cette manière, des grilles d'entretien en phase avec les réalités observées dans la mise en œuvre des métiers du vitiviniculteur $(\mathrm{n}=20)$ peuvent être élaborées. Ces grilles ont pour 
volonté de saisir l'ensemble des savoirs métiers (mise en culture de la vigne, lutte contre les maladies, méthodes de vinification...) et de comprendre également les différents raisonnements et bricolages mis en œuvre par les acteurs dans le cadre de leur profession puisque : "l'enquête par entretien est particulièrement pertinente lorsque l'on veut analyser le sens que les acteurs donnent à leurs pratiques, aux événements dont ils ont pu être les témoins actifs » (Blanchet \& Gotman, 2015, p. 24). Ainsi afin de collecter tout le matériel nécessaire à la réalisation de l'ontologie du cycle de vie de la vigne, trois types de matériaux sont collectés :

- Les savoirs théoriques et scientifiques clés concernant la culture de la vigne et la vinification : une quarantaine de documents mobilisés pendant la formation ou par les viticulteurs ont été retenus.

- Les observations de terrain permettant de détecter les phases clés de la vigne pendant tout le cycle de la vigne sur 9 mois, la phase de dormance a été exclue.

- Les entretiens avec des viticulteurs : 20 entretiens ont été réalisés.

Ce projet est donc ancré dans une logique d'acteurs avec un déploiement d'une méthodologie holistique indispensable du fait de la modification importante ces dernières années de la manière de produire liée à des enjeux de mondialisation et des enjeux de durabilité (Prévost, 2011, p. 50). L'interdisciplinarité assumée et la mobilisation de différentes méthodologies s'expliquent par la complexité de saisir certaines notions. Si l'on regarde par exemple du côté de la production, le « terroir » est aujourd'hui une notion mobilisatrice chez les acteurs, mais pour autant très difficile à « scientifiser ». Ainsi cette notion ne fait, par exemple, plus l'objet d'enseignement spécifique dans les formations d'agronome : "Cette notion présente la particularité de ne plus être enseignée dans les formations classiques d'agronomes en France, alors que l'idée de terroir se diffuse largement, tant dans l'activité agricole à l'international, en lien avec les indications géographiques des productions, que dans les démarches de développement local, en France, mais aussi sur le plan international (Barham, 2001) » 
(Prévost, 2011). Dans ce type de cas, les savoirs théoriques et scientifiques ne sont donc pas suffisants et les savoirs d'expérience sont alors essentiels pour saisir toute la complexité du métier.

\section{La création d'un corpus de référence pour traiter des savoirs théoriques et scientifiques de la viticulture}

À l'heure actuelle, il n'existe pas un outil unique permettant de reprendre pas à pas les différents éléments constitutifs du cycle de la vigne et de la vinification. Outre le cycle en tant que tel, il est également nécessaire de dégager les thématiques pouvant être utiles pour la constitution de l'ontologie, car pouvant avoir un impact sur le cycle de la vigne. Il a donc été indispensable, par la mobilisation de plusieurs ouvrages de référence mais également des sites internet largement utilisés par les professionnels $\left(\mathrm{BASF}^{12}\right.$ pour les maladies de la vigne par exemple ou bien encore le site de l'Institut de la Vigne et du $\operatorname{Vin}^{13}$ ), de constituer dans un premier temps, une veille bibliographique thématique. Ainsi 7 grandes thématiques ont été explorées lors de ce travail sur les savoirs théoriques et scientifiques avec la mobilisation de 40 documents de référence. Les documents de référence ont été retenus du fait 1. De leur mobilisation dans les formations pédagogiques, 2. De leur reconnaissance par des institutions scientifiques reconnues (ex. INRA ${ }^{14}$ ), 3. De leur usage par les professionnels. Les 7 thématiques explorées sont les suivantes :

- Pied de vigne (1 document de référence mobilisé)

- Maladies de la vigne (6 documents de référence)

- Ravageurs de la vigne (9 documents de référence)

- Risques climatiques (1 document de référence)

- Maladies physiologiques (4 documents de référence)

- Viniculture (11 documents de référence)

- Cycle de la vigne (8 documents de référence)

12 https://www.agro.basf.fr

$13 \mathrm{http} / / / \mathrm{www} . v i g n e v i n . c o m$

$14 \mathrm{http}: / /$ www.inra.fr 
Le cycle de vie de la vigne est caractérisé par deux grands cycles : le cycle végétatif de la vigne et le cycle reproducteur. Le cycle végétatif correspond à la production des rameaux et des feuilles ainsi qu'à l'entretien de ceux-ci. Ainsi les étapes qui le constituent sont : la dormance, le débourrement, les pleurs, l'arrêt de la croissance, l'aoûtement et la chute des feuilles Les étapes d'entretien de la vigne sont quant à elles : l'épamprage, l'ébourgeonnage, le rognage, le relevage, l'effeuillage, l'éclaircissage et la taille. En parallèle, le cycle reproducteur correspond aux étapes de fabrication des fleurs et des fruits et leur maturation. Les étapes qui le constituent sont les inflorescences visibles, la floraison, la nouaison, la véraison, la maturité et les vendanges. Tout ce travail de dépliage du cycle de la vigne a ainsi permis de construire une carte conceptuelle globale du cycle de la vigne (Figure 1) en reprenant chaque étape clé. Il s'agit alors de caractériser grâce à la littérature la terminologie professionnelle en vigueur.

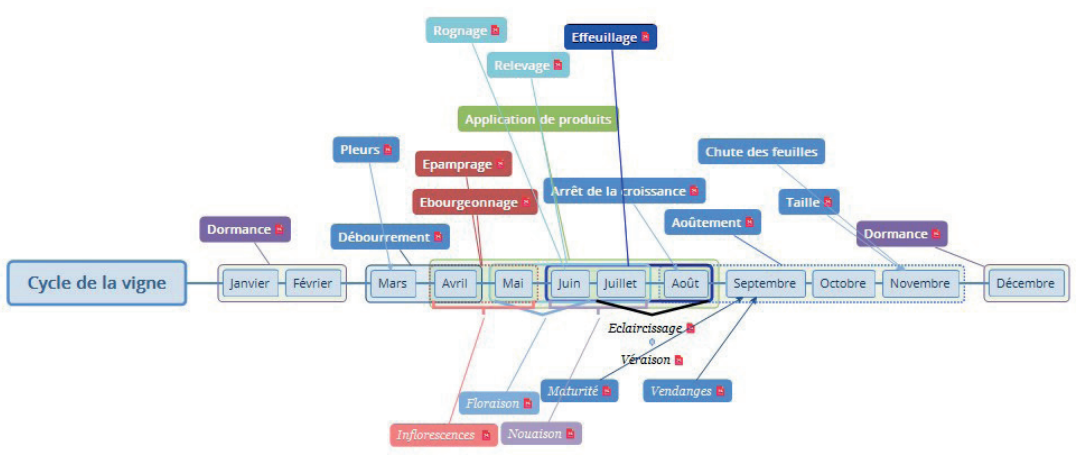

Figure 1. Carte conceptuelle du cycle de vie de la vigne $e^{15}$

Chaque thématique mise en évidence lors de ce cycle annuel de la vigne a fait l'objet d'un traitement spécifique avec pour objectif systématique de répondre aux questions suivantes afin ensuite de pouvoir se conformer aux règles logiques de constitution de l'ontologie : Quoi ? Quand ? Comment ? Pourquoi ? Les risques?

15 Pour une vision dynamique : http://winecloud.checksem.fr/cycle-vigne 


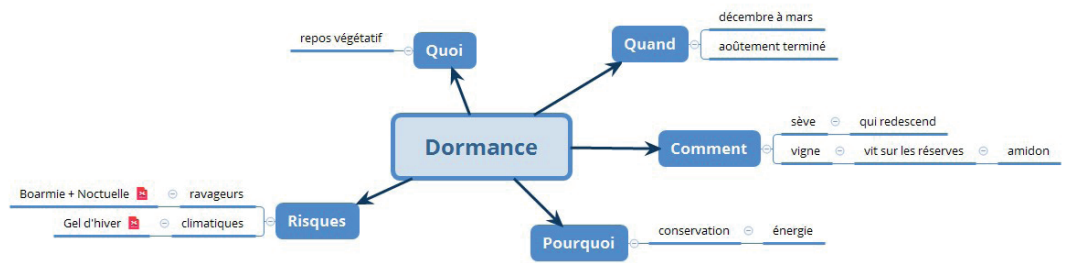

Figure 2. Carte conceptuelle de la dormance (cycle de la vigne) sur la base bibliographique

Ce travail de conceptualisation de la vigne a permis alors de développer 58 cartes conceptuelles (voir ci-dessous) en fonction des différentes thématiques relevées lors du travail de veille des savoirs théoriques et scientifiques.

- Cycle de la vigne, 20 cartes conceptuelles réalisées : aoûtement, arrêt de la croissance, couper les courants, débourrement, dormance, ébourgeonnage, éclaircissage, effeuillage, épamprage, floraison, inflorescence visible, maturité, nouaison, pied de vigne, pleurs, relevage, rognage, taille, vendange, véraison.

- Climat, 6 cartes conceptuelles réalisées : échaudage, excès d'eau, foudre, gel, grêle, vent.

- Nutrition, 8 cartes conceptuelles réalisées : azote, bore, carence azote, dessèchement, fer, folletage, magnésium, potassium.

- Ravageurs, 17 cartes conceptuelles réalisées : abeilles, acariens jaunes, acariens rouges, acariens, acariose, boarmie et noctuelle, cicadelle flavescence, cicadelle verte, cochenille, cochylis, erinose, eudémis, phylloxera, punaise verte, pyrale, ravageurs occasionnels, thrips.

- Vinification, 17 cartes conceptuelles réalisées : assemblage, bâtonnage, collage, débourbage, dégustation, égouttage, élevage du vin, encuvage, fermentation alcoolique, fermentation malo-lactique, mise en bouteille, pressurage, soutirage, sulfitage, vieillissement, vinification en blanc, vinification. 
- Maladie, 10 cartes conceptuelles réalisées : black-rot, bois noir, botrytis, esca, eutypiose, excorioise, flavescence dorée, mildiou, oïdium, rougeot.

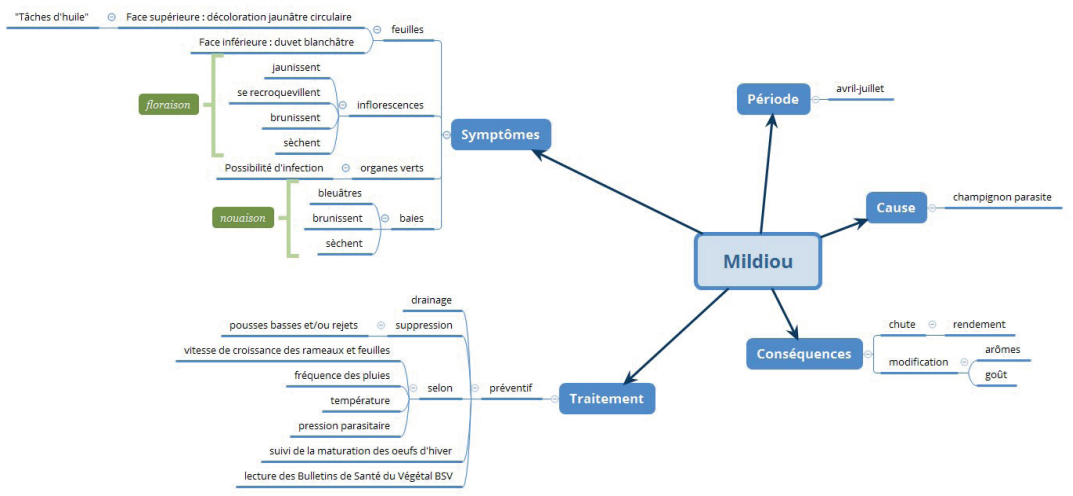

Figure 3. Carte conceptuelle du mildiou (maladie) sur la base bibliographique

Il est à noter que la question législative n'a pas été abordée comme sujet spécifique, mais apparaît toutefois de manière disparate dans l'ensemble du travail réalisé, la vitiviniculture faisant l'objet de nombreuses lois (cahier des charges, réglementations, AOC, etc.) qui définissent un certain nombre de pratiques. Du fait de la complexité du sujet exploré, il a été nécessaire de développer plusieurs grilles d'entretien afin de prendre en considération les différents paramètres venant impacter le cycle de la vigne. Ainsi, quatre grilles d'entretiens semi-directifs explorant différentes thématiques ont été développées :

- Cycle de la vigne

- Maladies

- Ravageurs

- Risques climatiques et physiologiques

Dans le cadre du projet, 20 entretiens avec des viticulteurs âgés de 30 à 62 ans d'une même zone géographique et travaillant pour le compte d'une même coopérative ont eu lieu (Saône-etLoire 71). Le travail exposé sera donc spécifique à cette coopé- 
rative, permettant d'avoir des données à la fois circonscrites et riches. Les entretiens réalisés sont à mi-chemin entre l'entretien semi-directif et l'entretien d'explicitation. En effet, il s'agit d'explorer l'ensemble des thématiques dégagées lors de la réalisation des cartes conceptuelles, tout en leur demandant de nous expliciter leurs vécus et leurs connaissances du terrain afin de donner une autre résonance à tous les savoirs mis en lumière lors du travail de recherche scientifique et théorique. Pour cela, un guide d'entretien a été réalisé au préalable afin de lister les points à aborder tout en laissant la liberté à l'enquêté de s'exprimer et de nous expliciter ses pratiques. L'entretien d'explicitation " est une technique d'aide à la verbalisation. Son utilisation se situe a posteriori, une fois l'activité qui fera l'objet de l'explicitation réalisée. Son objectif est de guider le sujet dans la verbalisation précise du déroulement de sa conduite, c'est-à-dire à la suite de ses actions matérielles et/ou mentales »(Vermersch, 1993, p. 63). Dans cette perspective, plusieurs types de questions sont posés visant à permettre une prise de recul réflexive sur les pratiques mises en œuvre et une conscientisation (Vermersch, 2004). Dans un premier temps, des questions définitoires ou liées à leurs connaissances initialement théoriques, mais qui ont pu se redéfinir selon les pratiques réelles (1). Pour les maladies, il s'agit par exemple de demander au viticulteur interviewé de nous définir selon ses propres mots et sa propre perception de différentes maladies : "qu'est-ce que la flavescence dorée ? " ou bien encore "qu'est-ce que le bois noir? " et des éléments de connaissance avec des questions du type : «quels sont les symptômes de la flavescence dorée? ». Dans un second temps des questions liées à leurs pratiques sur le terrain (2) avec des questions plutôt précises concernant les différentes maladies : "comment faut-il agir en cas de bois noir? 》 ou bien encore « avez-vous des astuces pour éviter d'avoir à utiliser beaucoup de produits phytosanitaires ? " sont posées. Et enfin, dans un troisième temps, ils étaient interrogés sur leur propre représentation en lien direct avec leurs pratiques (3) : " quelles sont les maladies les plus importantes/graves? ". 


\section{Des savoirs théoriques et scientifiques aux savoirs d'expérience des professionnels}

L'objectif de ces travaux de captation puis d'analyse des données n'est pas d'opposer les savoirs théoriques et scientifiques aux savoirs d'expérience et d'y introduire une classification subjective hiérarchique, mais de rendre compte grâce au travail d'explicitation comment ces savoirs cohabitent, s'enrichissent, se redéfinissent. En effet, comme le rapporte très justement Jacques Bonniel dès 1983 après avoir étudié l'articulation entre la science, le savoir et la culture de la viticulture : "la logique de développement de la science viticole n'est pas la simple application des découvertes de la chimie végétale ou de la biochimie : elle s'appuie sur un fonds de savoirs dont certains seront articulés àlet dans une pensée scientifique, mais à laquelle d'autres resteront irrémédiablement extérieurs " (Bonniel, 1983). Par ailleurs, dans une logique de la transmission des savoir-faire (Desbois-Thibault, 2003) il est essentiel de ne pas opposer les rapports entre savoir et sciences et la clé de compréhension se situe dans les pratiques culturelles de la population étudiée.

Les entretiens permettent de souligner que les stratégies mises en œuvre par les viticulteurs sur le terrain sont beaucoup régies par des paramètres législatifs et scientifiques. En effet, l'application de certains traitements est directement liée à des décrets (1) : « il y a des stades, donc on est prévenus, de telles dates à telles dates il faut absolument traiter et c'est obligatoire de toute façon les traitements puisque c'est un décret donc on les fait». Par ailleurs dans le cas de maladies, des prélèvements peuvent être faits en cas de doute afin de vérifier ou non une hypothèse : "C'est la Chambre d'Agriculture qui vient faire des prélèvements, dès qu'on a fini la prospection on marque les pieds, les rangs, on y marque sur une carte après pour qu'eux ils puissent se repérer, ils viennent repérer ça, ils prennent les feuilles et ils les emmènent à analyser ».

Ces savoirs scientifiques et législatifs qui contraignent les pratiques ou bien les confortent, s'ajustent également avec d'autres types de savoirs, notamment les savoirs d'expériences qui sont pluriels : les apprentissages académiques (2), les savoirs d'expé- 
rience des pairs (3), les savoirs d'interprétation du contexte, (dans le cas de la viticulture plus particulièrement, il s'agit le plus souvent de l'interprétation des paramètres météorologiques) (4) et les savoirs liés aux essais et aux bricolages (travail de la vigne de manière non formelle et non académique) qui ne sont pas forcément vérifiés par des études scientifiques (5).

\section{Tableau 1. Typologie des savoirs d'expérience en viticulture}

\begin{tabular}{|c|c|c|}
\hline & $\begin{array}{l}\text { Les savoirs } \\
\text { d'expérience } \\
\text { mobilisés }\end{array}$ & Verbatim \\
\hline 1 & $\begin{array}{l}\text { Adaptation des } \\
\text { normes et des } \\
\text { règles }\end{array}$ & $\begin{array}{l}\text { « il y a la ponte il faut jouer sur la ponte et éviter que l'in- } \\
\text { secte ponde. Et ça en fin de compte c'est la Fredon qui font } \\
\text { des comptages et c'est eux après qui nous envoient la date... } \\
\text { et la date vient de la préfecture donc c'est une obligation » } \\
\text { " aujourd'hui c'est obligatoire. C'est pareil c'est dans } \\
\text { le cahier des charges de la préfecture avec la flavescence } \\
\text { dès qu'un bois n'est pas aoûté bah faut l'arracher. » }\end{array}$ \\
\hline 2 & $\begin{array}{l}\text { Apprentissage } \\
\text { académique }\end{array}$ & $\begin{array}{l}\text { "alors la flavescence dorée, donc c'est... on va se rappeler } \\
\text { des cours... " } \\
\text { « enfin bon je ne savais même pas qu'il existait celui-là. } \\
\text { Mais moi c'est pareil les études hého il y a longtemps.» }\end{array}$ \\
\hline 3 & $\begin{array}{l}\text { Savoirs d'expé- } \\
\text { rience des pairs }\end{array}$ & $\begin{array}{l}\text { «le tonnerre, ça fait tourner la vendange ça a toujours été dit } \\
\text { depuis des générations que s'il tonne pendant les vendanges } \\
\text { et que la vendange est bien mûre, s'il pleut, s'il y a quand } \\
\text { même d'autres facteurs, il y a de gros risques de pourriture. } \\
\text { J'ai déjà connu des années où ça a pourri en } 72 \text { on a vu partir } \\
\text { la récolte. » } \\
\text { "Il y a des dictons : si les cerises sèchent sur le cerisier, les } \\
\text { raisins ne pourriront pas » }\end{array}$ \\
\hline 4 & $\begin{array}{l}\text { Connaissances du } \\
\text { terrain et interpré- } \\
\text { tation du contexte }\end{array}$ & $\begin{array}{l}\text { "non c'est le temps qui nous guide.» } \\
\text { "L'oïdium on sait pas trop comment il fait pour se } \\
\text { développer si vite, que le mildiou on sait qu'après une pluie, } \\
\text { un gros coup de chaleur qui est tout de suite derrière, il y } \\
\text { a de la rosée plein les feuilles, on sait que ça c'est un gros } \\
\text { risque de mildiou comme ça vient plus tard on est un peu } \\
\text { démuni quand on le voit» }\end{array}$ \\
\hline
\end{tabular}




\begin{tabular}{|c|c|l|}
\hline 5 & $\begin{array}{l}\text { «On essaie, là j'ai essayé du charbon de bois dans les rangs } \\
\text { de vigne, à première vue... » } \\
\text { «alors il y a des essais, il y a des charbons à mettre dans le } \\
\text { sol, pour essayer de capter les champignons, justement pour } \\
\text { pas qu'ils aillent dans le cep, il y a des produits à mettre } \\
\text { dessus, sur les plaies mais bon je ne connais pas de produit } \\
\text { miracle. On teste des boîtes à musique nous, aussi, sur l'es- } \\
\text { essais et brico- } \\
\text { lages }\end{array}$ \\
\hline
\end{tabular}

L'ensemble de ces savoirs permet tout d'abord d'apporter des éléments complémentaires aux savoirs théoriques par exemple pour détecter certaines maladies. Dans le cas du bois noir et de la flavescence dorée, les symptômes sont quasiment les mêmes et l'interprétation du viticulteur est indispensable : " c'est les mêmes symptômes que le bois noir mis à part que la flavescence, si réellement on a un doute, ça fait souvent des ronds. L'insecte, on va dire, se déplace très peu, donc il contamine souvent des ronds. Donc quand on voit un rond, qu'on a 5-6 pieds à un endroit, c'est un gros risque de flavescence dorée ».

Ces savoirs permettent par ailleurs d'interpréter une situation et de mettre les éléments en perspective : "L'Esca c'est souvent, alors, c'est les gros coups de chaleur, les gros écarts de températures et souvent on passe la veille il est vert, bien, et deux jours après on voit qu'il commence à être rabougris et c'est fini, pour lui c'est fini. Là, cette année, il n'y en a pas eu de trop parce qu'on a eu de la chaleur, mais c'est resté assez constant. Ce n'était pas des gros pics et donc on n'en a pas trop, trop eu, on en a bien assez quand même parce que ça occupe déjà. Mais on en a un petit peu moins que d'habitude ce qui va être une bonne chose, ça va nous faire une petite bouffée d'oxygène. Mais c'est vrai que cette maladie, c'est sournois parce qu'on ne la voit pas arriver ».

Ainsi tout le travail de veille des savoirs académiques est ici mis en écho grâce aux entretiens conduits avec les viticulteurs permettant de mieux saisir les savoirs dans toute leur complexité et de comprendre leurs constructions. Ceci a notamment permis d'enrichir les cartes conceptuelles afin de peupler l'ontologie et de souligner que les savoirs métiers amènent une somme d'information essentielle afin de saisir toute la complexité de la pratique. Un recoupement entre les données a donc été nécessaire afin de 


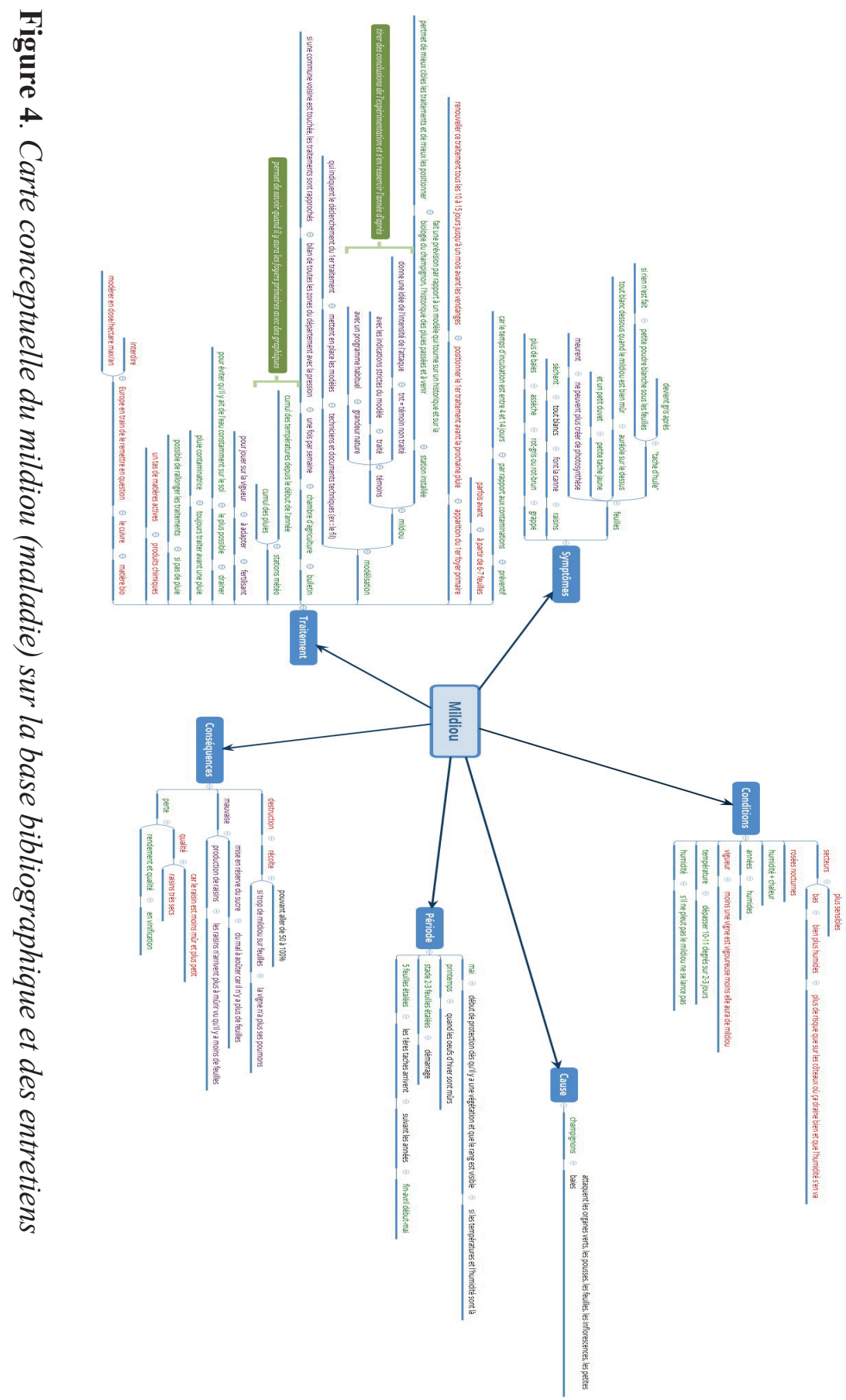


rendre compte de ce qui était présent dans les deux veilles (bibliographique et entretiens), de ce qui était uniquement présent dans la veille bibliographique mais pas appliqué par les viticulteurs pour diverses raisons, de ce qui était uniquement présent dans les entretiens et pas dans la veille bibliographique (essais, bricolages).

Le travail réalisé permet de souligner que les pratiques sont conditionnées par les savoirs académiques (théoriques, scientifiques et législatifs) mais également les savoirs d'expérience de nature protéiforme. L'ensemble de ces savoirs permet d'interpréter les situations, d'ajuster et d'affiner les pratiques selon tout un ensemble de données contextuelles. Ce travail souligne alors l'importance de se situer dans une logique écologique et pragmatique au sens de prendre en compte les réalités de terrain (Barbier et al., 2013), les expériences individuelles et collectives (Streith \& De Gaultier, 2012 ; Petit \& Barataud, 2015) pour donner une réalité contextuelle pratique à notre ontologie.

\section{Éléments de conclusion}

Comme souligné par Richardson : "les savoirs experts en agriculture ont peu à peu délégitimé d'autres formes de savoirs détenus par les agriculteurs eux-mêmes, souvent basés sur des masses d'observations et d'expériences faites dans un milieu spécifique et transmises de génération en génération " (Richardson, 2005). Les savoirs d'expériences mis en avant dans cette étude répondent bien à cette définition puisqu'ils sont de natures plurielles, à savoir : les savoirs d'expériences transmis par les pairs, les savoirs issus de l'interprétation du contexte et de la connaissance de leurs parcelles et les savoirs liés aux différents essais et solutions peu formels et scientifiques mis en place pour pallier les risques pouvant survenir au cours du cycle de la vigne (maladies, ravageurs, risques climatiques et physiologiques). Ces savoirs se complètent avec les savoirs théoriques et scientifiques, que ce soit par le respect des normes et décret ou par l'application de ce qu'ils ont pu apprendre au cours de leur apprentissage académique du métier de viticulteur. Pour autant, la création d'une ontologie spécifique exige qu'un protocole méthodologique pluridisciplinaire 


\section{alliant ici les Sciences de l'Information et la Communication et l'Informatique soit mis en place afin de traiter toutes les formes de savoirs sans hiérarchie. Cette condition est nécessaire dans le but de se rapprocher le plus près possible des savoirs du domaine métier et de créer un système logique en adéquation. L'ensemble de ce travail permet alors de souligner comment les savoirs s'enri- chissent, s'opposent et se redéfinissent.}

\section{Références}

Barbier, M., Cauchard, L., Joly, P.-B., Paradeisen, C. \& Vinck, D. (2013). Pour une Approche pragmatique, écologique et politique de l'expertise, Revue d'anthropologie des connaissances 7(1), 1-23.

Barham, E. (2001). Translating "terroir": social movement appropriation of French concept. Communication au workshop International perspectives on alternative agrifoods networks : quality, embeddedness, and bio-politics, Santa Cruz, 12-13 octobre.

Blanchet, A. \& Gotman, A. (2015, 2éd.). L'entretien. Paris : Armand Colin.

Bonniel, J. (1983). La transmission et la transformation des savoirs en milieu vigneron. Les savoirs naturalistes populaires 1, 23-30.

Davallon, J. (2003). La médiation : La communication en procès ? Dans M. Thonon (Éd.), Médiations et médiateurs. Coll. MEI. Médiation et Information 19 (pp. 3759). Paris : L'Harmattan.

Desbois-Thibault, Cl. (2003). L'extraordinaire aventure au champagne : Moët et Chandon une affaire de famille. Paris, Presses universitaires de France.

Jeanneret, Y. (2008). Penser la trivialité. Volume A : la vie triviale des êtres culturels. Coll. Communication, médiation et construits sociaux. Paris : Éd. Hermès-Lavoisier.

Malinowski, B. (1963). Les argonautes du pacifique occidental. Traduit de l'anglais par André et Simone Devyver. Préface de Sir James Frazer. Paris : Gallimard.

Minsky, M. (1974). A framework for representing knowledge, Artificial Intelligence $\mathrm{n}^{\circ}$ 306, Ed. Massachusetts Institute of Technology.

Petit, S. \& Barataud, F. (2015). L'eau source de savoirs : analyse de situations dans des bassins versants agricoles. Vertigo $15,1$.

Prévost, P. (2011). Enjeux didactiques dans la formation des agronomes : cas de la notion de terroir. Natures sciences et sociétés 19, 50-55.

Quillian, M. R. (1968). Semantic memory. Dans M. Minsky (Éd.), Semantic information processing (pp. 227-270). Cambridge MA : MIT Press.

Richardson, M. (2005). À la recherche de savoirs perdus ? Expérience, innovation et savoirs incorporés chez des agriculteurs biologiques au Québec. Vertigo 6, 1.

Sowa, J. F. (1984). Conceptual Structures: Information Processing in Mind and Machines. Boston MA : Addison-Wesley Longman Publishing Co.

Streith, M. \& De Gaultier, F. (2012). La construction collective des savoirs en agriculture bio : modèle pour l'agroécologie ? Dans D. Van Dam, M. Streith, J. Nizet \& P. Stassart (Éds). Agroécologie. Entre pratiques et sciences sociales (pp. 203-218). Dijon : Éducagri Éditions. 
Studer, R., Benjamins, V. R. \& Fensel, D. (1998). Knowledge engineering: principles and methods. Data and knowledge engineering 25(1), 161-198.

Sylvestre, J.-P. (1997). Apprentissage et transmission des savoirs et savoir-faire agricoles : ruses, bricolages et braconnages. Dans M. Guillemin (Éd.), Colloque « Agriculture et patrimoine : une dynamique pour l'agriculture » (pp. 59-74). LEGTA de Besançon, Dannemarie-sur-Crête.

Vermersch, P. (1993). L'entretien d'explicitation. Les cahiers de Beaumont 52bis-53, 63-70.

Vermersch, P. (2004). Aide à l'explicitation et retour réflexif. Éducation permanente $160,71-80$.

\section{Remerciements}

Nous remercions chaleureusement les caves de Lugny et les viticulteurs pour leurs concours précieux. Nous remercions l'ensemble des partenaires du projet : Vitagora, Orange, Photon Line, R-Tech Solutions, Images et Réseaux. Les auteur·es remercient Sébastien Gérin et Nicolas Gros pour leurs précieux concours au projet. 


\section{(c) (1) $\Theta($}

«Attribution - Pas d'Utilisation Commerciale - Pas de Modification 4.0 International» (CC BY-NC-ND) 